Antonie van Leeuwenhoek (2006) 89: 209

(C) Springer 2006

DOI $10.1007 / \mathrm{s} 10482-006-9075-9$

Erratum

Short communication

\title{
Erratum to: Isolation and sequencing of a new glucoamylase gene from an Aspergillus niger aggregate strain (DSM 823) molecularly classified as Aspergillus tubingensis
}

\author{
Friederike Manger-Jacob, Tobias Müller, Martina Janssen, Milan Höfer and Udo \\ Hölker* \\ Institut für Zelluläre und Molekuläre Botanik, Rheinische Friedrich-Wilhelms Universität Bonn, Kirschallee 1, \\ D-53115 Bonn, Germany; *Author for correspondence (e-mail: u.hoelker@uni-bonn.de; phone: +49-228- \\ 735510; fax: + 49-228-735504)
}

Published in Antonie van Leeuwenhoek, Volume 88, Nos. 3-4, pp. 267-275, 2005,

DOI 10.1007/s10482-005-3989-5.

Due to an unfortunate turn of events the sentences indicated below in the second column on page 272 were typeset erroneous. The correct version displayed below in the right hand column should however be treated as definitive by the reader.

The predicted molecular mass is $68.013 \mathrm{kDa}$, a value comparable with other Aspergillus glucoamylases, e.g., from A. shirousami with $68.126 \mathrm{kDa}$ (Acc. no. D10460) and A. kawachii with $68.267 \mathrm{kDa}$ (Acc. no. D00427), which are taxa belonging to the $A$. niger aggregate (Raper and Fennell 1965), and $A$. niger glucoamylase G1 with $65.424 \mathrm{kDa}$ (Acc. no. X00712). The amino acid sequence offers 8 asparagine linked glycosylation sites allowing an increase of the protein molecular mass by about $9 \mathrm{kDa}$ to approximately $77 \mathrm{kDa}$ (Ftouhi-Paquin et al. 1997). A similar increase in molecular weight is known from glucoamylase G1 from A. niger, which amounts after glycosylation to $82 \mathrm{kDa}$ (Svensson et al. 1983). Comparing sequence similarities among glucoamylases from several Aspergilli, that from $A$. tubingensis did show $92 \%$ similarity to $A$. niger G1 glucoamylase at the nucleotide level $99 \%$ and $94 \%$ at the amino acid levels. Its similarity with glucoamylases from $A$. shirousami and A. kawachii was at the nucleotide level. Converting these results into a phylogram (Figure 2) the data demonstrate that $A$. tubingensis glucoamylase belongs to the monophyletic group of $A$. kawachii, $A$. shirousami and A. awamori glucoamylases, which is distinctly different from the $A$. niger glucoamylase.

Within the five conserved regions identified in fungal glucoamylases, several amino acids were

Comparing sequence similarities among glucoamylases from several Aspergilli, that from $A$. tubingensis did show $92 \%$ similarity to $A$. niger $\mathrm{G} 1$ glucoamylase at the nucleotide level and $94 \%$ at the amino acid level. Its similarity with glucoamylases from A. shirousami and A. kawachii was $99 \%$ at the nucleotide level. 